\title{
Branch-Like Iron Nitride and Carbide Magnetic Fibres via Electrospinning Technique
}

\author{
A. García-Márquez ${ }^{*[a]}$, S. Glatzel ${ }^{[b]}$, A. Kraupner ${ }^{[c]}$, K. Kiefer ${ }^{[d]}$, K. Siemensmeyer ${ }^{[d]}$ and C. Giordano* ${ }^{[e]}$
}

Abstract: $\mathrm{Fe}_{3} \mathrm{~N}$ and $\mathrm{Fe}_{3} \mathrm{C}$ nanocomposites have a wide range of applications thanks to their ceramic nature, magnetic properties, conductivity and catalytic activity, just to cite some. In many fields optimal performances are ensured by crystallinity, homogeneity and hierarchical organization. In the present paper, crystalline, magnetic and well-defined nanofibres of iron nitride and iron carbide/carbon nanocomposite with tunable composition and size were prepared via electrospinning. The starting polymeric material was directly electrospun into fibres and then calcined, leading to a highly homogeneous final product of nanoparticles along the fibres (both outside and inside). A mechanistic study was undertaken and here discussed. The magnetic properties of the as-prepared nanofibres were also studied. The as-prepared final fibre mat composite material can serve as active catalyst, e.g. in oxygen reduction reaction (where nanofibres outperformed mere nanoparticles), it can serve as functional support for classical catalytic processes or, thanks to its magnetic properties, can be applied in magnetic-field assisted separation or as magneto-active membranes.

\section{Introduction}

Iron is one of the most abundant elements on earth and is present in nature in many essential compounds, such as hemoglobin, but also in artificial materials highly relevant to industrial processes, e.g steel. One of its most interesting properties is its magnetism, as pure element or in chemical compounds formed with oxygen, nitrogen or carbon. Some oxides, such as magnetite and maghemite, nitrides (in a wide form of crystalline polymorphs e.g. $\alpha, \beta, \varepsilon$ ), or cementite (iron carbide) are among the most magnetic species reported, besides elemental iron ${ }^{[1]}$. Whereas iron oxide nanoparticles have been widely studied and applied, e.g. in hyperthermia treatments ${ }^{[2]}$, as specific drug delivery mediators ${ }^{[3]}$ or as contrast agents in magnetic resonance imaging (MRI) ${ }^{[4]}$, novel alternatives have recently been proposed in order to overcome the drawback of a comparably low saturation

[a] Dr. A. García-Márquez, Facultad de Química, Universidad Nacional Autónoma de México Av. Insurgentes Sur 3000, Ciudad Universitaria, C.P. 10200, Mexico City, Mexico. agarciam@unam.mx

[b] Dr. S. Glatzel, School of Chemistry, University of Glasgow, Glasgow, G12 8QQ, UK

[c] Dr. A. Kraupner, NanoPETPharma GmbH, Luisencarrée, RobertKoch-Platz 4, 10115 Berlin, Germany.

[d] Dr. K. Kiefer and Dr. K. Siemensmeyer Helmholtz-Zentrum Berlin für Materialien und Energie, 14109 Berlin, Germany

[e] Dr. C. Giordano, Max Planck Institute of Colloids and Interfaces, Colloid Department, Am Mühlenberg 1, 14476 Potsdam, Germany. Current address: School of Biological and Chemical Sciences, Queen Mary University of London, Mile End Road, London E1 4NS, UK. c.giordano@qmul.ac.uk

Supporting information for this article is given via a link at the end of the document. magnetization. Thus, iron nitrides and carbides were pointed out as promising alternatives for their high saturation magnetization ${ }^{[5]}$ and their superior tensile strength and hardness ${ }^{[6]}$.

From a synthetic point of view, synthesis of $\mathrm{Fe}_{3} \mathrm{~N}$ and $\mathrm{Fe}_{3} \mathrm{C}$ presents the same challenges as other metal nitrides and carbides, with a further issue: high reaction temperature can bring to their decomposition into $\mathrm{Fe}^{\circ}$ and $\mathrm{N}_{2} / \mathrm{C}$. Originally, iron carbide phases were only found as side products in some processes, such as graphitization of carbon, in the presence of iron-based catalysts. More recent syntheses are flame spray pyrolysis $\left(\mathrm{Fe}_{3} \mathrm{C}\right)$, CVD or ammonolysis of presynthesized iron or iron oxide nanoparticles ${ }^{[7]}$. Most of these methods are energy consuming, not ideal for large scale production and, to the best of our knowledge, not suitable for morphological control.

In order to fully utilize the properties of iron nitride and carbide materials, the processing method becomes fundamental aspect. Dealing with fibres rather than unstructured materials can also bring further advantages, such as catalytic selectivity and improved electron transport ${ }^{[8,9]}$. However, iron carbide and nitrides, as many metallic ceramics, are very difficult to mold and process after their synthesis. Therefore, the possibility to shape them upon preparation, keeping morphology and properties, is a significant advantage. The easily pre-synthesis processing of the iron based fibres allows, for instance, to create neat, yet porous, membranes keeping electron transport but also for applications as magnetic sensors, filters or shielding, in magnetic cell separation or as contrast agents in MRI. If the nanofibres are also organic/inorganic hybrids, a novel multifunctional material is obtained, whose properties of the metallic ceramic, such as thermal and chemical resistance, magnetism and catalytic activity can overlap those of the organic part (e.g. porosity, light weight). In this respect, it is important that the filling NPs are uniformly distributed inside the hosting matrix, avoiding agglomeration during the synthesis.

Electrospinning is a relatively simple, yet versatile technique for preparing fibres with diameters within the micrometer till the nanometer scale, in large scale. The versatility of this technique allows the production of a variety of complex organized structures such as ribbons, core-sheath composites $^{[10]}$, hollow tubes ${ }^{[11]}$ or hierarchically confined nanoparticles within multilayered fibres (e.g. iron oxide nanoparticles in core-polyethylene/shell-polystyrene obtained via mulitaxial electrospinning ${ }^{[12]}$ ).

However, despite its extensive use in the field of polymer science, not many examples report electrospun fibres of inorganic or hybrid systems have been reported. Some examples include $\mathrm{ZnO}^{[13-15]}, \mathrm{TiO}_{2}{ }^{[16,17]}, \mathrm{FeO}_{x}{ }^{[8,9]}$ and $\mathrm{PtFe}$ 
based organic/inorganic fibres ${ }^{[18]}$. References concerning non-oxide materials, such as silicon carbide fibres ${ }^{[19]}$, superconductive chevrel phase sulfides ${ }^{[20]}$ and titanium nitrides and oxynitrides ${ }^{[21]}$ are uncommon due to difficulty of the synthetic procedure.

Our group has designed novel pathways to overcome the problems related to the synthesis of metal nitrides and carbides by a novel sol-gel process (the urea-glass-route ${ }^{[22]}$ ), by employing biopolymers ${ }^{[5]}$ or heterocyclic molecules ${ }^{[23]}$, which allows the preparation of a wide variety of MN/MC in relatively mild, but also greener and safer conditions ${ }^{[24]}$. The present work describes a simple, inexpensive, and versatile route to obtain hierarchical nanostructured iron nitride and carbide composite fibres via an improved polymeric urea glass route, previously designed for the production of chromium nitride and carbide fibres ${ }^{[25]}$. The polymeric moiety acts as both urea-like species and fibre scaffold, which also minimizes the number of waste coproducts.

The final magnetic nanofibres may have applications as diverse as data storage device, magnetic filters and sensors. The supported-particle mat could be used as membrane supported catalyst, and can represent a greener, low-cost and less toxic alternative to tantalum ${ }^{[26]}$ and ruthenium ${ }^{[27]}$ catalysts, with a further advantage to be magnetic and therefore easily recoverable. Finally, one of the most recent and important applications of iron/nitrogen/carbon based nanofibres is as promising catalysts for ORR in Proton Exchange Membrane Fuel Cell (PEMFC) ${ }^{[8,18,28,2]}$, which offers a clean process for energy conversion. Classically employed catalysts are based on expensive platinum, which is easily poisoned during the process, whilst are outperformed, in some cases by Fe-N-Cs (or Fe-N-C based materials) ${ }^{[8,18,28]}$. More interesting, it was observed that the catalyst performance towards ORR is strongly related to the synthetic process and structure of the catalysts (in particular as ribbons and fibres), which show higher activity than mere randomly distributed $\mathrm{NPs}^{[8,9]}$. However, most of these studies deal in fact with a mixed "Fe-N-C" catalyst. This lack of definition in composition makes difficult to indubitably identify the active phase and thus improving current performances. In order to understand the reason for the activity and fully exploit the properties of these materials as catalyst in ORR and catalytic reactions in general, the first important step is to prepare stable and well-defined catalysts (both in composition and shape). In this respect, the processing method becomes a fundamental aspect.

In our final systems, the carbide or the nitride phase are welldefined in shape, as well as chemical composition, highly crystalline and uniformly distributed in the functional carbon matrix, all key requisites for a good catalytic performance but above all to identify and understand the role of the active catalysts. Compared to other similar synthesis, we do not prepare the carbon fibres ahead and then impregnate them with the iron salt, we start rather with a polymer solution containing the iron, the nitrogen and the carbon precursors, which is electrospun directly into fibres and then calcined, leading to a more homogeneous final product.

\section{Results and Discussion}

For the design of the fibre system, it was necessary to choose a fibre source and a suitable iron precursor both able to form homogeneous solutions. A previous study using polymers required a resin as an additive, which was an inconvenience due to the multiple parameters to be controlled at once ${ }^{[25]}$. By profiting of a polymer that possesses a complexing character, high molecular weight and acts as well as a nitride source, metal nitride and carbides fibres can be easily obtained by electrospinning by employing a minimum number of components. In the present study, we have found poly( $N$-vinylpyrrolidone) (PVP) to be the most suitable polymer for the preparation of this ideal polymeric precursor. Iron (II) acetylacetonate was chosen as metal precursor because of its higher stability towards iron alcoholates formation ${ }^{[30]}$. In fact, acetylacetonate has been previously used as a capping ligand for metal alcoxides formation for other species, such as titanium ${ }^{[31]}$. In addition, the high molecular weight PVP presented high compatibility with the iron precursor due to its capability for cation complexation. The iron to PVP ratio used for electrospinning was a 1:1 mass ratio. Increased mass content of iron(II)acetylacetonate resulted in the precipitation from the solution. Methanol proved to be a good solvent for either substance, with further advantages, such as a rapid evaporation rate and a higher dielectric constant, compared for instance, to ethanol.

To optimize the synthetic route and maximize the material qualities, a systematic electrospinning study was performed, investigating the influence of the collector-to-tip distance, the solution flow rate and the applied voltage. The distances-to collector tested were 8 and $12 \mathrm{~cm}$; the applied voltages were 10,14 and $18 \mathrm{kV}$ and the injection flows were $0.25,0.50$ and $1.0 \mathrm{~mL} / \mathrm{h}$. The humidity was kept constant at $25 \%$.

The employed system proved to be very stable given that bead-free fibres were obtained at any of the electrospinning conditions probed. Nonetheless, we observed dripping at lower voltages. The optimal processing conditions were: a voltage of $14 \mathrm{kV}$, needle-to-collector distance of $12 \mathrm{~cm}$ and flow rate of $0.25 \mathrm{~mL} / \mathrm{h}$. These conditions yielded bimodal fibres with two average diameters (of $80 \mathrm{~nm}$ and of $300 \mathrm{~nm}$ respectively). By decreasing the voltage, the two previously observed fibre diameters underwent into a broader diameter distribution ranging from $200 \mathrm{~nm}$ to $1 \mu \mathrm{m}$. This loss of the two average populations was also observed for the fibres electrospun at higher flow rates. Some of the known 
parameters that affect fibre shape, size and morphology are the polymer weight, the polymer concentration ${ }^{[32]}$, the solvent ${ }^{[33]}$ and the relative humidity ${ }^{[34]}$. Similar observations were reported for electrospun fibre yarns with bimodal diameter distributions of one order of magnitude difference ${ }^{[35,36]}$. The results suggest that the jet stability is strongly affected by the humidity. Different studies ${ }^{[25,37]}$ also justified coinciding observations as a consequence of secondary jets arising from the initial one.

Further analysis of the polymer size and polydispersity (PDI) were performed by gel permeation chromatography (GPC). The molecular weight of the polymer presented two average molecular weight populations (Mw) of 170000 and 750000 Da (Figure S1, Supplementary Information). From the previous GPC data and the electrospinning results, this bimodality of the fibre diameter derives somehow from the two Mw observed. Moreover, the conductivity of the solution is high due to both the methanol and the iron (II) acetylacetonate, which explains the absence of beads even at low voltages. The formation mechanism of our fibres resides in the following observations. At controlled humidity, the bimodal fibre formation is a function of the instabilities of the jet polymer. Thus, at higher voltages, the jet will be stable enough to form a minimum of secondary jets with welldefined diameters producing the two average fibre sizes. This model remains limited to our systems and, to the authors' knowledge, it is the first time that the bimodality of a polymer is kept after the processing technique.

\section{Influence of the dwelling temperature.}

The nonwoven mats were stabilized under air at $280^{\circ} \mathrm{C}$ for 2 h. This process fixed the fibre conformation for further thermal treatment. The omission of this curing step yielded molten structures.

Analysis of SEM micrographs (Figure 1a and c) indicates that the two fibre diameter populations are preserved after the stabilization process and that the diameters of both population of fibres decreased by approximately $50 \%$ (Figures $1 \mathrm{~b}$ and $\mathrm{d}$ ).

This decrease is attributed to either the evaporation of residual solvent absorbed in the fibres or a partial decomposition of the polymeric scaffold. The iron content was determined by inductively coupled plasma atomic emission spectrometer (ICP-AES). Iron content was found to be $37 \%$. Additionally, powder X-ray diffraction (XRD) after the stabilization step did not reveal any crystalline structure (Figure 7, $280^{\circ} \mathrm{C}$ ).

Further thermal treatment of the fibres was carried out under nitrogen atmosphere at different temperatures ranging from 400 to $700{ }^{\circ} \mathrm{C}$. The heating ramp was $2 \mathrm{~h}$ and the dwelling time was fixed at $1 \mathrm{~h}$. The resulting products were black fibre mat structures with a brittle aspect and were stable at room temperature.

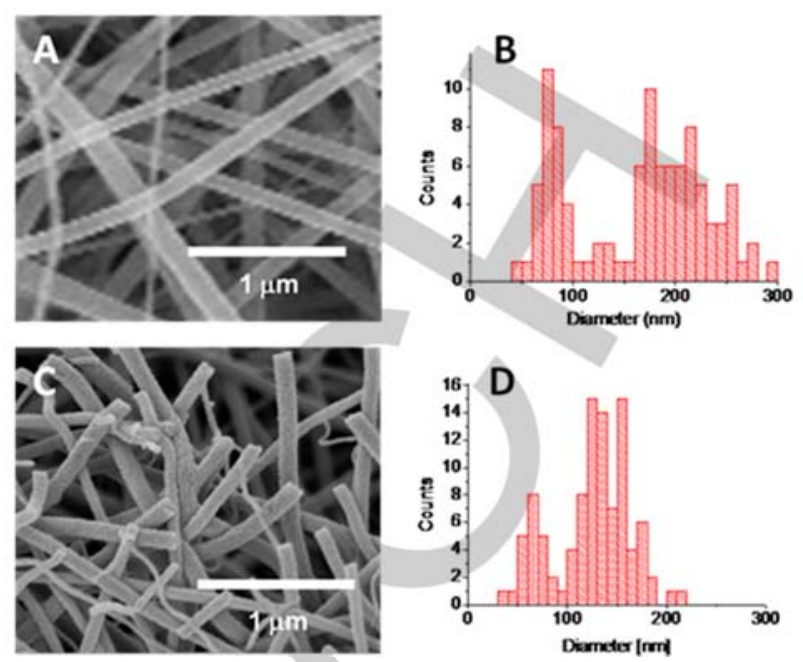

Figure 1. SEM images of the precursor fibres a) as spun and c) after the stabilization step at $280^{\circ} \mathrm{C}$. Histograms of the corresponding fibre diameters are shown in b) and d) respectively. The diameters were obtained over a population of at least 50 fibres.

Elemental analysis of the products as a function of temperature indicated a decrease of the carbon content from 39 to $20 \mathrm{wt} \%$ and an increasing percentage of the relative iron content, determined by ICP-AES, ranging from 36 to 41 $w t \%$. The carbon decrease can be explained as a progressive decomposition of the polymer scaffold as a function of temperature, thus the increasing relative iron content is due to a loss of carbon, hydrogen and oxygen as a result of decomposition of the fibre scaffold. It can also safely assumed that the major part of carbon is leaving as $\mathrm{CO}_{\mathrm{x}}$. These outcomes are schematically reported in figure 2 .
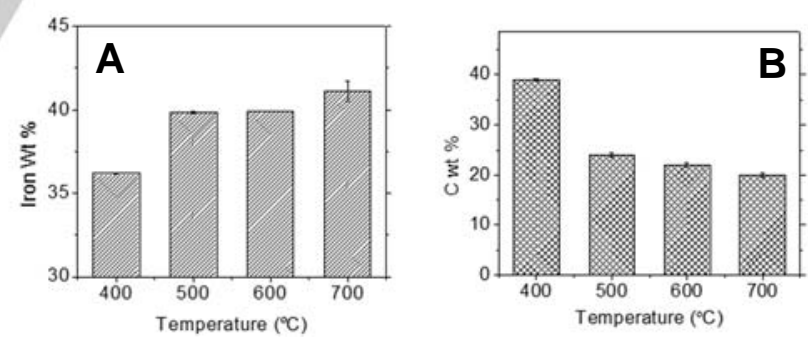

Figure 2. Mass percentage of a) iron and b) carbon present in fibres treated at different temperatures. 
Figure 3 reports the XRD patterns of iron oxide, iron nitride and iron carbide containing electrospun fibres treated at different temperatures.

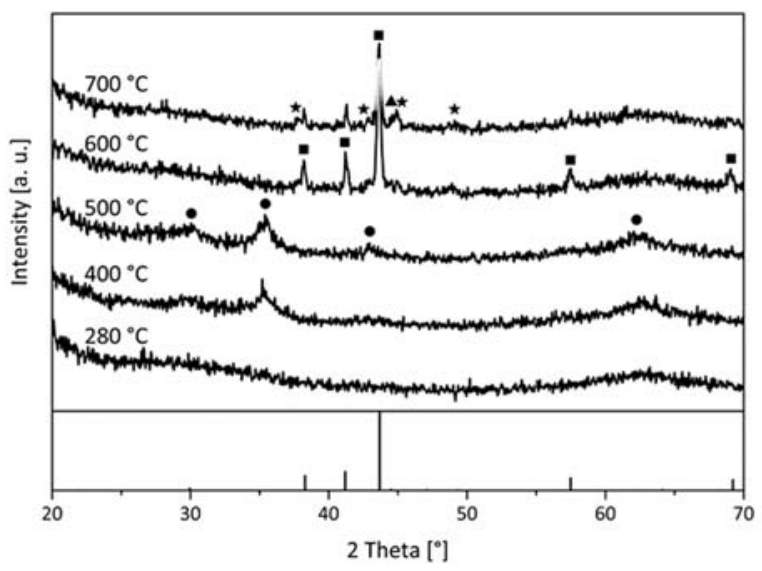

Figure 3. XRD patterns of iron oxide (circles), iron nitride (squares) and iron carbide (stars) containing electrospun fibres treated at different temperatures. Ref. Pattern (full vertical line): iron nitride (ICCD04-007-2250).

This figure shows that the first crystalline iron species were observed at $400^{\circ} \mathrm{C}$ and correspond to iron oxide $\left(\mathrm{Fe}_{3} \mathrm{O}_{4}\right)$, with only the corresponding planes (311) and (440) observed. At $500^{\circ} \mathrm{C}$ $\mathrm{Fe}_{3} \mathrm{O}_{4}$ is still the only species present, but the corresponding XRD pattern shows sharper reflections. Additionally, a reflection at $2 \theta=$ $42^{\circ}$, corresponding to the (400) plane can be observed. This pattern is in good agreement with previously reported polymer$\mathrm{Fe}_{3} \mathrm{O}_{4}$ nanoparticle composite fibres ${ }^{[38]}$. At $600^{\circ} \mathrm{C}$ the complete conversion of the iron oxide into nitride occurs and $\mathrm{Fe}_{3} \mathrm{~N}$ is the only crystalline phase present, with an average correlation distance of $30 \mathrm{~nm}$. Finally, elemental iron and iron carbide were observed on fibres treated at $700^{\circ} \mathrm{C}$.

No crystalline carbonaceous species were observed in the XRD spectra in figure 3 (due to the absence of any peak around $26^{\circ}$ ), indicating that the iron species do not act as graphitization catalysts.

In Figure 4, SEM images of the non-woven mats are shown. These images indicate a correlation of the change in surface texture of the fibres and the dwelling temperature. The roughness of the fibre surface increased gradually over the whole temperature range. At $600{ }^{\circ} \mathrm{C}$ particles with an average diameter of $60 \mathrm{~nm}$ were observed on the surface of the fibres (Figure 4c), which is in good agreement with the crystallite sizes calculated from the X-ray diffraction patterns. Despite the observed wrinkled texture, the nitrogen sorption studies have shown a moderate surface area of approximately $20 \mathrm{~m}^{2} / \mathrm{g}$.

These branch-like mats suggested that the iron nitride particles grew from the inside of the fibre structure. Based on previous observations ${ }^{[39]}$ we suggest that the oxide to nitride transformation is a local surface nitridation between the nitrogen from both the polymer and the reaction atmosphere.
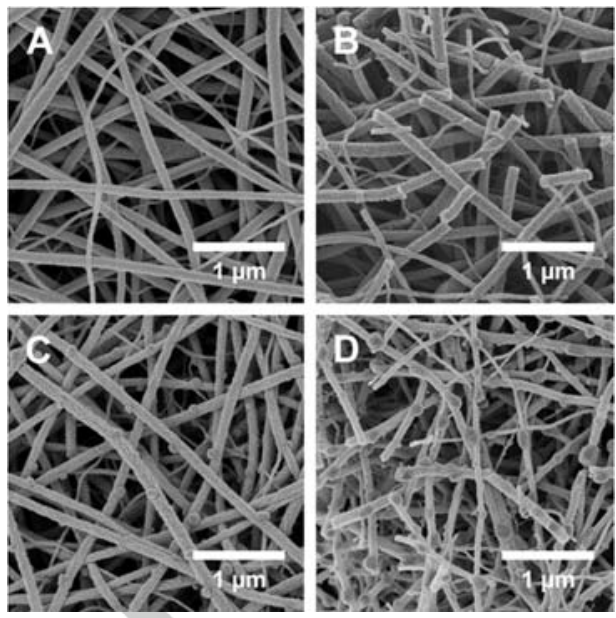

Figure 4. SEM images of samples treated at a) $400{ }^{\circ} \mathrm{C}$, b) $500{ }^{\circ} \mathrm{C}$, c) $600{ }^{\circ} \mathrm{C}$ and d) $700^{\circ} \mathrm{C}$

Further characterization via TEM of the temperature treated fibres was performed to have a better understanding of the nitride formation mechanism.

TEM study on the fibres calcined at $500^{\circ} \mathrm{C}$ showed well-defined structures (see Figure 5) and also revealed small particles of $c a$. $2 \mathrm{~nm}$ deposited on a carbonaceous scaffold. The size of the iron particles is in good agreement with the particle sizes estimated from the corresponding XRD pattern via Scherrer equation ${ }^{[40]}$.
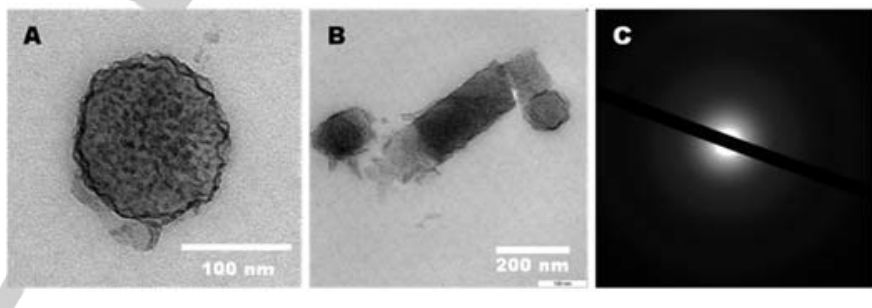

Figure 5. TEM images of the fibres treated at $500^{\circ} \mathrm{C}$ : a) cross sectional, b) lateral structure and c) corresponding SAED image.

Kalra et al. observed similar patterns in dispersed magnetite particles into block copolymer-silica core-shell fibre systems ${ }^{[12]}$. Piperno et al. also reported the growth of silver nanoparticles within a polyacrylonitrile scaffold ${ }^{[41]}$. TEM micrographs of fibres calcined at $600^{\circ} \mathrm{C}$ are shown in Figure 6. These studies showed nanoparticles with larger sizes, between 20 and $30 \mathrm{~nm}$, embedded into the carbon fibre scaffold. The formation of aggregates was rarely observed, suggesting that the particle nucleation takes part at specific points of the polymer. SAED studies showed the planes (211) and (111) of iron nitride particles. No additional planes corresponding to the planes (002) and (010) of graphite were observed, indicating that the fibre scaffold is made of amorphous carbon (as also indicated by the XRD pattern). 
The ultramicrotomy of these fibres indicates that iron nitride species are both outside and inside the particles (figure 6).

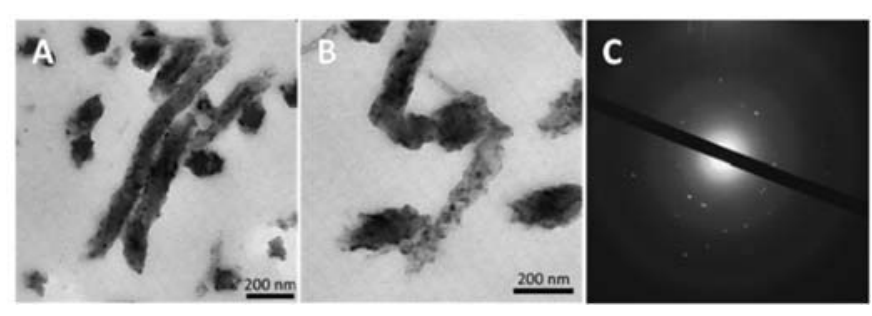

Figure 6. a) TEM image showing both lateral and cross sectional parts of the fibres; b) TEM ultramicrotomy studies of fibres treated at $600^{\circ} \mathrm{C}$ with c) SAED of the previous image.

\section{Influence of the dwelling time.}

In order to gain control over the particle size of the iron nitride and to observe its transition into iron carbide and elemental iron, the influence of the dwelling time was also studied at $600^{\circ} \mathrm{C}$ and 700 ${ }^{\circ} \mathrm{C}$ between 0 and $2 \mathrm{~h}$.

Dwelling time at $600^{\circ} \mathrm{C} . \mathrm{XRD}$ studies of the thermally treated fibres did not show any crystalline species below $1 \mathrm{~h}$ of dwelling time, evidencing a slow crystallization rate of iron nitride, which is the kinetic product. Moreover, a slight increase of the crystallite size on the sample with a dwell of $2 \mathrm{~h}$ was observed. XRD diffractograms for a $2 \mathrm{~h}$ dwelling time sample also shown an additional reflection corresponding to the (300) plane (Figure 7A).
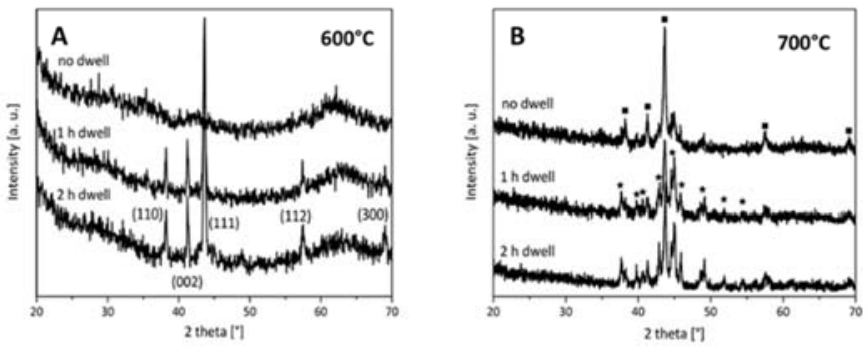

Figure 7. $X R D$ pattern of iron nitride (squares) and iron carbide (stars) containing fibre mats calcined at $600^{\circ} \mathrm{C}(\mathrm{A})$ and $700^{\circ} \mathrm{C}(\mathrm{B})$ with different dwelling times

Dwelling time at $700^{\circ} \mathrm{C} . \mathrm{XRD}$ diffractograms of samples treated at $700{ }^{\circ} \mathrm{C}$ with different dwelling times (Figure 7B) show the formation of iron nitride and traces of iron carbide in fibres without dwelling time. Moreover, the intensity of the signal of iron carbide increases with increasing the dwelling time, while the intensity of the iron nitride peaks decreases and broadens, suggesting a partial transformation of the nitride into carbide by simple reaction of the iron with the fibre scaffold, in line with previous observations ${ }^{[39]}$. Elemental iron was also found in traces in any sample and it increases as a function of the dwelling time as previously observed by our group ${ }^{1}$.
The magnetic properties of fibres calcined at $500^{\circ} \mathrm{C}$ and $600^{\circ} \mathrm{C}$ were determined by means of a Superconducting Quantum Interference Device (SQUID) magnetometer with QuantumDesign MPMS-5T at $300 \mathrm{~K}$.

Magnetization curves of the sample calcined at $500{ }^{\circ} \mathrm{C}$ seen in Figure 8 a, exhibit a typical sigmoidal behaviour with a slight hysteresis, corresponding to a simple superparamagnetic species. This can be explained in terms of the particle size and composition and it is comparable with analogue size $\mathrm{Fe}_{3} \mathrm{O}_{4}$ nanoparticles ${ }^{[35]}$. Similar results were observed on iron oxide fibre composites bearing particles ranging from 5-15 $\mathrm{nm}^{[41]}$.

The magnetization plot for the fibres treated at $600^{\circ} \mathrm{C}$ and 2 hours of dwelling time (Figure $8 \mathrm{~b}$ ) showed ferromagnetic behaviour as expected for iron nitride. The saturation value of $122 \mathrm{emu} / \mathrm{g}_{\mathrm{Fe}}$ and a coercitivity value $\mathrm{H}_{\mathrm{C}}=112 \mathrm{~T} / \mathrm{g}_{\mathrm{Fe}}$ and a remanence $\mathrm{B}_{\mathrm{R}}=10.4$ $\mathrm{emu} / \mathrm{g}_{\mathrm{Fe}}$. This value is slightly lower than those obtained for thin films obtained by treatment of iron nitride thin layers ${ }^{[36]}$. We rationalize this in terms of the system shape, seen as iron nitride particles dispersed into a matrix.
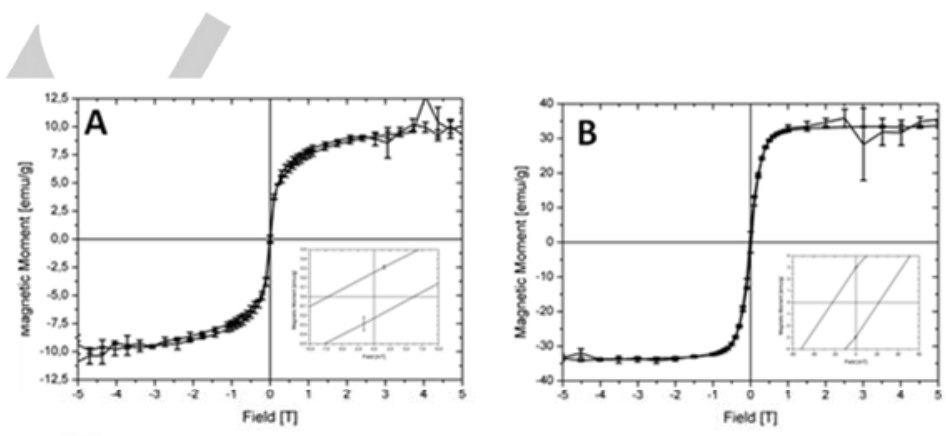

Figure 8. Magnetization plots of fibres treated at a) $500^{\circ} \mathrm{C}$ and b) $600^{\circ} \mathrm{C}$.

\section{Conclusions}

In the present study we report a robust and versatile strategy for the synthesis and easy processing of submicrometric to nanometric fibre made of iron nitride/carbide carbon nanocomposites prepared via nitrogen jacketed electrospinning. Thermal treatment of the mats above 600 ${ }^{\circ} \mathrm{C}$ yielded iron nitride nanostructures with branch-like morphology, as result of a surface nitridation of the original iron species. Composition, crystallinity and magnetic properties of the mats can be controlled by temperature and dwelling time. To the best of our knowledge this is the first time that iron nitride and carbide submicron and nanometer sized fibres are prepared with defined composition and crystallinity. The manifold properties of the iron nitride and carbide fibres allow their potential application in catalysis, ORR, magnetic separation and as magneto-active membranes. 


\section{Experimental Section}

Sample preparation. For a typical synthesis $250 \mathrm{mg}$ of iron(II)acetylacetonate (99.95\% trace metals basis, Aldrich) was mixed with $200 \mathrm{mg}$ poly(vinylpyrrolidone) - K 90 (powder, average $\mathrm{Mw} 360,000$, Fluka) and dissolved in $2 \mathrm{~g}$ of methanol. Approximately $0.6 \mathrm{~mL}$ of the resulting solution was drawn into a $1 \mathrm{~mL}$ syringe and the syringe was placed in the electrospinning device. The fibres were spun at an acceleration voltage of $14 \mathrm{kV}$, a solution feed-rate of $0.5 \mathrm{~mL} / \mathrm{min}$ and a tip - collector distance of $12 \mathrm{~cm}$.

The resulting fibre mats were stabilized in an oven under air. The heating rate was $2.8 \mathrm{~K} / \mathrm{min}$ to a temperature of $280{ }^{\circ} \mathrm{C}$ and the sample was held at that temperature for two hours. The stabilized mats were subsequently calcined at $600{ }^{\circ} \mathrm{C}$ under nitrogen atmosphere. The heating rate under nitrogen was $10 \mathrm{~K} / \mathrm{min}$ and the sample was held again at the desired temperature for two hours.

Characterization techniques. Samples were characterized by XRD measurements, performed on a Bruker D8 diffractometer using with $\mathrm{Cu}$-Ka radiation $(\lambda=0.154 \mathrm{~nm})$ and a scintillation counter. Nitrogen sorption experiments were done with a Quantachrome Quadrasorb at liquid nitrogen temperature, and data analysis was performed by Quantachrome software. All the samples were previously degassed at $150{ }^{\circ} \mathrm{C}$ for 20 hours before measurements. Elemental analysis was done for carbon, hydrogen and nitrogen using a Vario EL Elementar. Iron content was determined by ICP-OES technique using a Vista-MPX CCD Simultaneous ICP-OES with radial plasma. TEM images were taken using a Zeiss EM $912 \Omega$ operated at an acceleration voltage of $120 \mathrm{kV}$. Samples were either ground and then suspended in ethanol or embedded in LR White Resin (Medium Grade, Agar Scientific) and cut with a Leica Ultracut UTC to yield ultramicrotomes of the samples. One drop of the aforementioned suspension or an ultramicrotome was put on a 400 mesh carboncoated copper grid and left to air to dry or was used as is. SEM was performed on a LEO 1550 Gemini instrument. The samples were loaded on carbon coated stubs and coated by sputtering an $\mathrm{Au} / \mathrm{Pd}$ alloy prior to imaging. TGA measurements were performed under nitrogen between 50 and $1000{ }^{\circ} \mathrm{C}$ (heating rate $10 \mathrm{~K} / \mathrm{min}$ ) with a TG 209 F1 Iris, Netzsch. Magnetic properties were measured in a SQUID magnetometer (Quantum Design MPMS5T) equipped with a 5T superconducting magnet. Molecular weight and molecular weight distribution of the commercial PVP has been determined by size exclusion chromatography (SEC) performed at $70^{\circ} \mathrm{C}$ in dimethyl sulfoxide (DMSO) containing $5 \mathrm{~g} / \mathrm{L}$ of $\mathrm{LiBr}$ as eluent, using a pre-column and one PSS-GRAL-LIN analytical column (particle size $10 \mu \mathrm{m}$ ) with a flow rate of 1 $\mathrm{mL} / \mathrm{min}$. The employed detectors were a RI- (Shodex RI-71) and a UV-Detector (Spectra-System UV 1000; 300 nm). For calibration, linear poly(methyl methacrylate) standards (PSS, Germany) were used.
Magnetic Properties. The magnetic properties of the specimens were determined with a MPMS-5T Magnetic Properties Measurement System (Superconducting Quantum Interference Device, SQUID), by Quantum Design. For a typical measurement approximately $2 \mathrm{mg}$ of sample were placed into a gel capsule and pressed in firmly. The capsule was placed into the machine and the applied field was slowly raised to $5 \mathrm{~T}$ in order to assure magnetic saturation of the sample (measurement of the initial magnetisation curve). The field was then lowered back to $-5 \mathrm{~T}$ and raised again to $5 \mathrm{~T}$ to measure the saturation curves. After that zero field cooling and field cooling curves were measured at 500 $\mathrm{mT}, 1 \mathrm{~T}$ and $5 \mathrm{~T}$ fields.

\section{Acknowledgements}

The authors would like to thank Ms. Rona Pitschke for transmission electron microscopy, Marlies Gräwert for GPC measurements, Silvia Pirok for elemental analysis, Katharina Otte and Zoe Schnepp for SEM images, the Laboratory for Magnetic Measurements at the Helmholtz-Zentrum Berlin for SQUID measurements and the Max Planck Society for funding.

Keywords: iron nitride • iron carbide • fibres • electrospinning • sol-gel

[1] C. Giordano, A. Kraupner, S. C. Wimbush and M. Antonietti, Small, 2010, 6, 1859-1862.

[2] J.-H. Lee, J. Jang, J. Choi, S. H. Moon, S. Noh, J. Kim, J.-G. Kim, I.-S Kim, K. I. Park and J. Cheon, Nat. Nanotechnol., 2011, 6, 418-422.

[3] R. Asmatulu, M. A. Zalich, R. O. Claus and J. S. Riffle, J. Magn. Magn. Mater., 2005, 292, 108-119.

[4] J. M. Perez, L. Josephson, T. O'Loughlin, D. Högemann and R. Weissleder, Nat. Biotechnol., 2002, 20, 816-820.

[5] Z. Schnepp, S. C. Wimbush, M. Antonietti and C. Giordano, Chem Mater., 2010, 22, 5340-5344.

[6] J. Wang and Y. Zhou, Annu. Rev. Mater. Res., 2009, 39, 415-443.

[7] a) A. K. Schaper, H. Hou, A. Greiner, and F. Phillipp, J. Catal., 2004, 222, 250-254. b) I. K. Herrmann, R. N. Grass, D. Mazunin, and W. J. Stark, Chem. Mater., 2009, 21, 3275-3281. c) Y. Koltypin, X. Cao, R. Prozorov, J. Balogh, D. Kaptas, and A. Gedanken, J. Mater. Chem., 1997, 7, 24532456. d) R. S. Ningthoujam and N. S. Gajbhiye, Mater. Res. Bull., 2008, 43, 1079-1085.

[8] X. Yan, K. Liu, X. Wang, T. Wang, J. Luo and J. Zhu, Nanotechnology, 2015, 26, 165401

[9] X. Yan, L. Gan, Y.-C. Lin, L. Bai, T. Wang, X. Wang, J. Luo and J. Zhu, Small, 2014, 10, 4072-4079.

[10] J. T. McCann, D. Li and Y. Xia, J. Mater. Chem., 2005, 15, 735.

[11] H. Xiang, Y. Long, X. Yu, X. Zhang, N. Zhao and J. Xu, CrystEngComm, 2011, 13, 4856.

[12] V. Kalra, J. H. Lee, J. H. Park, M. Marquez and Y. L. Joo, Small, 2009, 5, 2323-2332.

[13] R. Nain, M. Jassal and A. K. Agrawal, Compos. Sci. Technol., 2013, 86, 9-17.

[14] X. Sui, C. Shao and Y. Liu, Polymer, 2007, 48, 1459-1463.

[15] S. Anitha, B. Brabu, D. J. Thiruvadigal, C. Gopalakrishnan and T. S. Natarajan, Carbohydr. Polym., 2012, 87, 1065-1072.

[16] S. Kedem, J. Schmidt, Y. Paz and Y. Cohen, Langmuir, 2005, 21, 5600 5604 .

[17] A. E. Deniz, A. Celebioglu, F. Kayaci and T. Uyar, Mater. Chem. Phys., 2011, 129, 701-704.

[18] G. Panthi, M. Park, H.-Y. Kim and S.-J. Park, J. Ind. Eng. Chem., 2015 24, 1-13. 
[19] H. Ye, N. Titchenal, Y. Gogotsi and F. Ko, Adv. Mater., 2005, 17, 15311535.

[20] K. V. Woan, R. H. Scheffler, N. S. Bell and W. M. Sigmund, J. Mater. Chem., 2011, 21, 8537.

[21] M. Zukalova, J. Prochazka, Z. Bastl, J. Duchoslav, L. Rubacek, D. Havlicek and L. Kavan, Chem. Mater., 2010, 22, 4045-4055.

[22] C. Giordano, C. Erpen, W. Yao, B. Milke and M. Antonietti, Chem. Mater. 2009, 21, 5136-5144.

[23] A. Kraupner, Markus Antonietti, R. Palkovits, K. Schlicht and C. Giordano, J. Mater. Chem., 2010, 20,6019.

[24] C. Giordano and M. Antonietti, Nano Today, 2011, 6, 366-380.

[25] A. García-Márquez, D. Portehault and C. Giordano, J. Mater. Chem., 2011, 21, 2136.

[26] C. Yue, M. Trudeau and D. Antonelli, Chem. Commun., 2006, 1918.

[27] B. Askevold, J. T. Nieto, S. Tussupbayev, M. Diefenbach, E. Herdtweck, M. C. Holthausen and S. Schneider, Nat. Chem., 2011, 3, 532-537.

[28] N. Wu, Y. Wang, Y. Lei, B. Wang, C. Han, Y. Gou, Q. Shi and D. Fang, Sci. Rep., 2015, 5, 17396.

[29] M. Nie, P. K. Shen, M. Wu, Z. Wei and H. Meng, J. Power Sources, 2006, $162,173-176$

[30] J. Zha and H. Roggendorf, Adv. Mater., 1991, 3, 522-522.

[31] U. Schubert, Chem. Soc. Rev., 2011, 40, 575.
[32] S.-Y. Tsou, H.-S. Lin and C. Wang, Polymer, 2011, 52, 3127-3136.

[33] A. Celebioglu and T. Uyar, Mater. Lett., 2011, 65, 2291-2294.

[34] R. Gentsch, B. Boysen, A. Lankenau and H. G. Boerner, Macromol. Rapid Commun., 2010, 31, 59-64.

[35] S. Sun, H. Zeng, D. B. Robinson, S. Raoux, P. M. Rice, S. X. Wang and G. Li, J. Am. Chem. Soc., 2004, 126, 273-279.

[36] H. Naganuma, Y. Endo, R. Nakatani, Y. Kawamura and M. Yamamoto, Sci. Technol. Adv. Mater., 2004, 5, 83-87.

[37] A. Holzmeister, M. Rudisile, A. Greiner and J. H. Wendorff, Eur. Polym. J., 2007, 43, 4859-4867.

[38] D. Zhang, A. B. Karki, D. Rutman, D. P. Young, A. Wang, D. Cocke, T. H. Ho and Z. Guo, Polymer, 2009, 50, 4189-4198.

[39] Z. Schnepp, M. Thomas, S. Glatzel, K. Schlichte, R. Palkovits and C. Giordano, J. Mater. Chem., 2011, 21, 17760.

[40] Scherrer P., Nachrichten Von Koniglicher Ges. Wiss. Zu Gottingen Math.-Phys. Kl., 1918, 2, 96-100.

[41] S. Piperno, L. A. Gheber, P. Canton, A. Pich, G. Dvorakova and A. Biffis, Polymer, 2009, 50, 6193-6197. 


\section{WILEY-VCH}

\section{Entry for the Table of Contents}

FULL PAPER

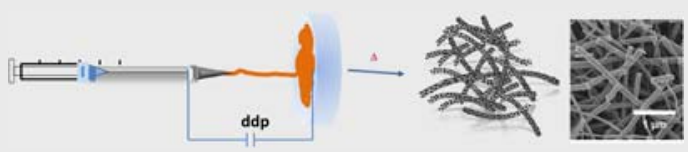

Electrospinning joins sol-gel process for the easy synthesis of ideal $\mathrm{Fe}_{3} \mathrm{~N}-\mathrm{Fe}_{3} \mathrm{C} / \mathrm{C}$ magnetic fibres with full control over composition, size and crystallinity.
A.García-Márquez*, S. Glatzel, A. Kraupner, K. Kiefer, K. Siemensmeyer and C. Giordano*

Page No. - Page No.

Branch-Like Iron Nitride and Carbide Magnetic Fibres via Electrospinning Technique 